\title{
Podoplanin expression in cancer-associated fibroblasts correlates with VEGF-C expression in cancer cells of invasive ductal breast carcinoma
}

\author{
B. PULA ${ }^{1,2}$, A. WOJNAR ${ }^{3}$, W. WITKIEWICZ ${ }^{1}$, P. DZIEGIEL ${ }^{1,2,4}$, M. PODHORSKA-OKOLOW $^{1,2, *}$
}

${ }^{1}$ Regional Specialist Hospital, Research and Development Centre, Wroclaw, Poland; ${ }^{2}$ Department of Histology and Embryology, Medical University, Wroclaw; ${ }^{3}$ Lower Silesian Oncology Centre, Wroclaw; ${ }^{4}$ Department of Physiotherapy, Wroclaw University School of Physical Education, Wroclaw, Poland

*Correspondence: marzenna.podhorska-okolow@umed.wroc.pl

Received October 27, 2012/ Accepted February 7, 2013

\begin{abstract}
Podoplanin (D2-40) was shown to be expressed in cancer-associated fibroblasts (CAFs) of various malignancies. The study aimed at examining its impact on angiogenesis and lymphangiogenesis markers in invasive ductal carcinoma of the breast (IDC). The studies were performed on 104 archival cases of IDC using immunohistochemical technique. Podoplanin expression in CAFs correlated positively with cancer cell VEGF-C expression $(r=0.19, p=0.0495)$ and intratumoral microvessel count $(\mathrm{MVC})$ of CD31 positive vessels $(\mathrm{r}=0.30, \mathrm{p}=0.0018)$, whereas negative correlations were observed with peritumoral MVC of D2-40 and Lyve- 1 positive lymphatic vessels $(r=-0.26, p=0.008$ and $r=-0.27, p=0.0058$, respectively). Podoplanin expression in CAFs did not correlate with VEGF-A and VEGF-D expression in cancer cells, nor exerted any prognostic significance. Podoplanin expression in CAFs may have impact on angio- and lymphangiogenesis processes in IDC.
\end{abstract}

Key words: breast cancer, podoplanin, VEGF-C, stroma, cancer-associated fibroblasts

Recent advances in cancer research in past decade led to new discoveries highlighting the complexity of various malignancies [1]. Although, the process of tumor vascularization stems from the pioneering work of Judah Folkman since the 70 's, it remains a crucial problem in cancer management [1, 2]. Tumor angiogensis and lymphangiogenesis were identified as key processes in tumor progression, enabling oxygen and nutrient supply, therefore allowing cancer cell growth and metastatic dissemination [1].

Numerous lines of evidence suggest, that these processes may be strongly affected by tumor stroma composed, in addition to cancer cells, of cancer-associated fibroblasts (CAFs), endothelial cells, inflammatory cells and components of the extracellular matrix $[1,3,4]$. CAFs were shown to promote tumor growth and angiogenesis by recruitment of endothelial progenitor cells via secretion of stromal derived factor-1 (SDF1) [5]. CAFs alter the tumor microenvironment by secreting matrix metalloproteinases (MMPs), which promote cancer cell growth and invasiveness $[6,7]$. Moreover, CAFs secrete various cytokines, such as transforming growth factor $\beta$ (TGF $\beta$ ), hepatocyte growth factor (HGF), epidermal growth factor
(EGF) and vascular endothelial growth factor A (VEGF-A), affecting cancer cell biology $[3,5,8]$.

Podoplanin (D2-40), first identified as a specific marker of lymphatic endothelium, was recently shown to be expressed in CAFs of various cancer types affecting patients clinical outcome in majority of the studies [9-17]. In addition, this highly $\mathrm{O}$-glycosilated, mucin-type transmembrane glycoprotein is expressed in various cancer cells, where depending on cell type, influences cell growth and invasiveness [18-22]. Moreover, it is suggested that it participates in the epithelial-mesenchymal transition (EMT), which may be the source of CAFs $[8,23,24]$. Recent study of Suzuki et al. revealed, that podoplanin expression in lung squamous cancer cells decreases the expression levels of VEGF-C and attenuates lymphangiogenic metastasis of these cells by reducing lymphatic vessel densities [25].

Although, podoplanin is rarely expressed in cancer cells of invasive ductal breast carcinoma (IDC), its expression is often noted in CAFs, which were shown to promote tumor angioand lymphangiogensis $[5,12,13]$. Therefore, in this study we aimed at examining the impact of podoplanin expression in CAFs on angio- and lymphangiogenetic processes of IDC. 


\section{Material and methods}

Patients. The studies were performed on 104 IDC cases, sampled in the years 1999-2002 during resection and biopsy procedures in the Lower Silesian Oncology Centre in Wroclaw. The clinical and pathological data were obtained from the archives of the Lower Silesia Oncology Centre in Wroclaw (Table 1). Patients age ranged from 30 to 83 years (mean 55.9 \pm 11.6 years), all the patients were female. All tissue specimens used in this study were collected before the beginning of the treatment. In total 87 (83.7\%) patients had undergone Patey's mammectomy and 17 (18.3\%) quadrantectomy followed by subsequent lymph node resection. Each patient was treated with suitable adjuvant therapy, according to the stage of the disease. In cases of stage 3 and higher, neoadjuvant therapy had been administered. In 70 (67.3\%) cases tamoxifen was administered. Radiotherapy was applied in 55 (52.9\%) cases The histological malignancy grade $(\mathrm{G})$ was determined using guidelines suggested by the criteria proposed by Elston and Ellis [26]. In the analyzed patients cohort $11(10.6 \%)$ cases were classified as G1, 57 (54.8\%) cases as G2 and 36 (34.6\%) cases as G3. The patients had been followed up for $59.3 \pm 38.8$ months (range $1-125$ months). In this period 20 (19.2\%) patients died of the disease.

Immunohistochemistry (IHC). Tissue samples were fixed in $10 \%$ buffered formalin, dehydratated and embedded in paraffin. Hematoxylin and eosin-stained $(\mathrm{H} \& \mathrm{E})$ preparations were done on all the samples. Immunohistochemical reactions were performed on $4-\mu \mathrm{m}$-thick paraffin sections in an automated staining platform Autostainer Link48 (Dako, Glostrup, Danemark). In order to deparaffinize, rehydrate and unmask the antigens the sections were boiled in Target Retrieval Solution buffer ( $\mathrm{pH}$ 9; Dako) using Pre-Treament Link Platform (Dako) and, subsequently, cooled in a rinsing buffer (TBS). Then, the sections were incubated with following murine primary antibodies directed against: podoplanin (D2-40 ready-to-use, RTU; Dako), CD31 (1:100, Leica Microsystems, Wetzlar, Germany) and VEGF-A (1:50, Dako). EnVision FLEX (Dako) was used to visualize the antigens. In the case of VEGF-A, incubation with primary antibody was extended from $20 \mathrm{~min}$ at room temperature (RT) to 18 hours at the temperature of $4^{\circ} \mathrm{C}$ and reaction was amplified using the EnVision $^{\text {not }}$ FLEX+ Mouse LINKER system (Dako).

In order to examine the expression of VEGF-C, VEGF-D and Lyve-1, murine antibodies directed against these antigens were utilized (all obtained in ReliaTech $\mathrm{GmbH}$, Braunschweig, Germany). Briefly, the sections were deparaffinized in xylene, rehydrated and boiled in a citrate buffer, pH 6 (for VEGF-C, Lyve-1) or pH 9 (for VEGF-D). Subsequently, activity of endogenous peroxidase was blocked by $5 \mathrm{~min}$ incubation in $3 \% \mathrm{H}_{2} \mathrm{O}_{2}$. The sections were incubated with primary antibody overnight at the temperature of $4^{\circ} \mathrm{C}(1: 100$, VEGF-C; $1: 100$, VEGF-D) or for $30 \mathrm{~min}$ at room temperature (1:50, Lyve-1). Then the antigens were visualized using the EnVision ${ }^{\text {th }}$ Detection Systems Peroxidase/DAB, Rabbit/Mouse (Dako).
Expression of estrogen (ER) and progesterone (PR) receptors were conducted according to the earlier described procedure [27]. The sections were deparaffinized and rehydrated in Antigen Retrieval Solution ( $\mathrm{pH}$ 6; Dako). Activity of endogenous peroxidase was blocked by $5 \mathrm{~min}$ incubation in $3 \% \mathrm{H}_{2} \mathrm{O}_{2}$. Subsequently, primary antibodies to ER (clone 1D5, 1:100) and PR (clone PgR 636, 1:100) and incubated for $1 \mathrm{~h}$ at RT. Following reaction stages were performed according to the manufacturer's instructions for the LSAB+ SystemHRP visualization system (Dako). 3,3'-diaminobenzidine (DAB) was used as a chromogen. HER-2 expression status was determined using the HercepTest ${ }^{\mathrm{tm}}$ kit (Dako), according to the procedure recommended by the producer. HER2 FISH pharmDx ${ }^{\text {mat }}$ Kit was utilized to determine the HER2 amplification status of cases which were scored equivocal $(+2)$ in the HercepTest ${ }^{\mathrm{tm}}$. All slides were counterstained with Mayer's haematoxylin (Dako). Subsequently, the preparations were mounted in SUB-X Mounting Medium (Dako).

Negative controls were performed by omitting the primary antibody, whereas tumor sections showing previously high expression of the analyzed marker were used as positive control. In case of podoplanin staining a positive reaction of lymphatic endothelial cells served as internal positive control of the staining.

Evaluation of IHC reactions. The IHC sections were evaluated under a BX-41 light microscope (Olympus, Tokyo, Japan). For podoplanin expression assessment in CAFs a semiquantitative immunoreactive score (IRS) method of Remmele and Stegner was utilized, which was successfully used for expression of markers in neoplastic cells [28]. The scale is originally based on the percentage of positive cells showing reaction in the whole section ( 0 pts: absence of cells with positive reaction, 1 pt: $1-10 \%$ cells, 2 pt: $11-50 \%, 3$ pt: $51-80 \%, 4$ pt: over $80 \%$ cells with positive reaction) and the intensity of the colour reaction ( 0 pts: no reaction, 1 pt: low intensity of the reaction product, $2 \mathrm{pt}$ : moderate intensity of the reaction colour, 3 pt: intense colour of the reaction). For the purpose of this study, the percentage of podoplanin positive cells was defined as clearly delineated podoplanin positive stromal area to the overall stromal area noted in the whole IDC tissue section (Figure 1). The final score represents the product of the two values, ranging within the scope of 0 to 12 .

The IRS method was also applied for the assessment of VEGF-A, VEGF-C and VEGF-D in IDC cancer cells. In this case, only cancer cells, but not the stromal area were taken into account. Utilization of the IRS scale for podoplanin assessment in CAFs and VEGFs expression in cancer cells would allow their direct comparison in the statistical analysis.

Chalkley Point Array graticule (Pyser Sgi., Edenbridge, UK) was utilized to determine the microvessel count (MVC) in all CD31, D2-40 and Lyve-1 stained sections. Three intratumoral and peritumoral hot-spots (areas of potentially the highest vascular density) were examined. Briefly, the sections were first scanned at low power (40x, 100x magnification) to identify the hot-spots and subsequently examined under $\times 200$ 


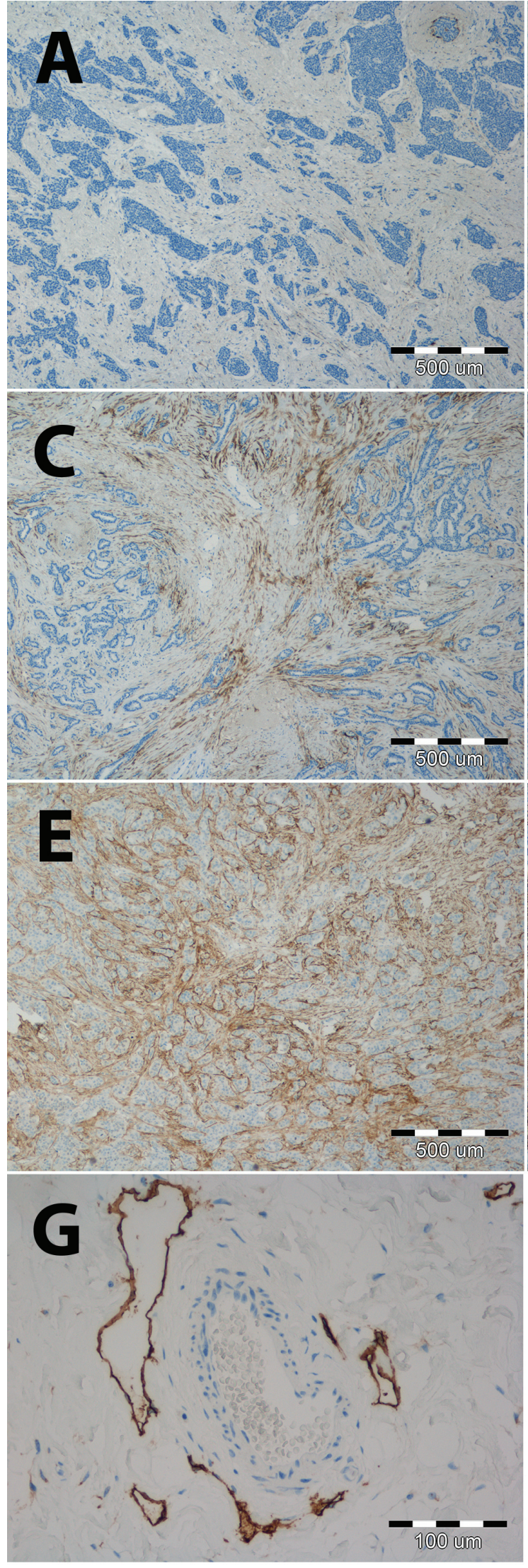

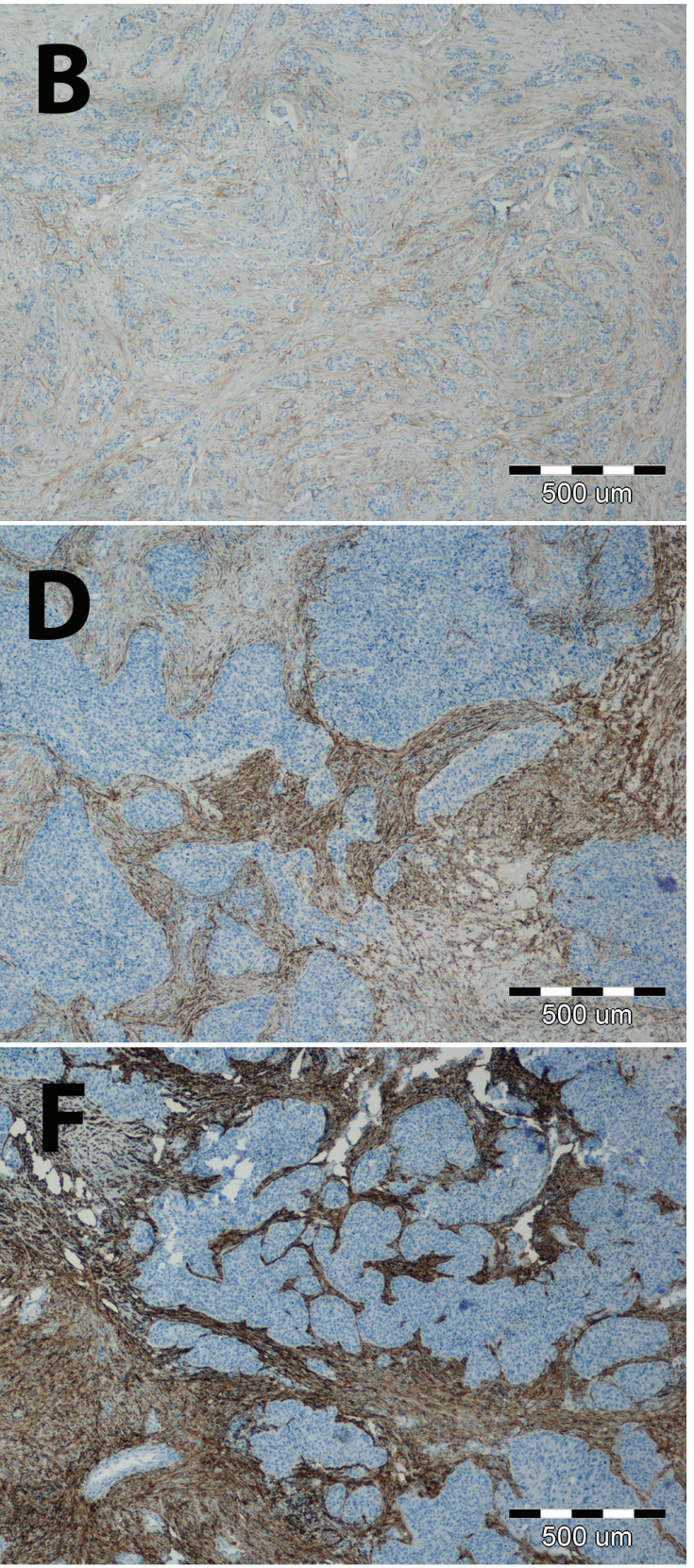

Figure 1. Differentiated expression of podoplanin in CAFs of IDC. Sample cases scored in the IRS scale as 0 pts. (A), 2 pts (B), 4 pts. (C), 6 pts. (D), 8 pts. (E) and 12 pts. (F). Strong podoplanin expression in lymphatic vessel endothelium served as positive internal control of the podoplanin stained sections (G). In contrast, no podoplanin expression is observed in blood vessel endothelium. Magnification $\times 40(A-F), \times 200(G)$. 
magnification using the point array. The Chalkley count was the number of grid points that hit stained microvessels [29]. Average score was determined for both intratumoral and peritumoral tumor compartments.

ER and PR expression assessment a four grade scoring system based on tumor cell positivity was utilized: $0(0 \%$ cells stained), 1 (1-10\% cells stained), 2 (11-50\% cells stained), 3 (51-100\% cells stained). ER and PR sections scored 1 and higher were regarded as positive according to the criteria of $11^{\text {th }}$ St. Gallen conference [30].

Statistical analysis. The results were analyzed using Prism 5.0 (GraphPad, La Jolla, CA, USA) and STATISTICA 8.0 (StatSoft, Krakow, Poland) software. The relationship between the expression of the studied markers was determined by Spearman's rank correlation. Relationship between podoplanin expression in CAFs and patients clinicopathological parameters were analyzed using Fisher's exact test. Overall survival (OS) was examined by the Kaplan-Meier method and the significance of the differences was determined by a log-rank test. Multivariate analysis was performed using the Cox proportional hazards model. The hazard ratio (HR) and $95 \%$ confidence interval $(95 \% \mathrm{CI})$ were estimated for each variable. The differences were accepted as significant at $\mathrm{p}<0.05$.

\section{Results}

Podoplanin expression in cytoplasm of spindle cells resembling the fibroblast-like phenotype of CAFs was noted in $82(82.7 \%)$ cases (Figure 1). Based on statistical analysis and our previous observations, the cases scored IRS 0-2 were regarded as podoplanin-low $(27 ; 25.9 \%)$, whereas these with IRS scores 3-12 were regarded as podoplanin-high $(77 ; 74.1 \%)$ [13]. When the categorized data were analyzed utilizing Fisher exact test, higher podoplanin expression correlated with larger primary tumor size $(\mathrm{p}=0.0271)$ and higher malignancy grade $(p=0.0006)$. No associations with patients age, menopausal status, presence of lymph node metastasis, ER PR and HER2 status were noted (Table 1).

VEGF-A, VEGF-C and VEGF-D expressions were noted in the cytoplasm of cancer cells, whereas CD31, D2-40 and Lyve1 were expressed in endothelial cells of corresponding blood (CD31) and lymphatic (D2-40 and Lyve-1) vessels. The cutoff values based on the medians after statistical analysis were determined for these markers and summarized in Table 2.

Spearman correlation rank test revealed, that podoplanin expression in CAFs showed a weak positive correlation with VEGF-C expression in cancer cells $(\mathrm{r}=0.19, \mathrm{p}=0.0495)$ and an average positive correlation with intratumoral CD31 MVC $(\mathrm{r}=0.30, \mathrm{p}=0.0018)$ (Figure 2). Analysis of serial sections immunostained for D2-40 and VEGF-C confirmed the results of statistical analysis (Figure 2). Moreover, podoplanin expression in CAFs correlated negatively with peritumoral D2-40 MVC $(\mathrm{r}=-0.26, \mathrm{p}=0.008)$ and peritumoral Lyve-1 MVC $(\mathrm{r}=-0.27$, $\mathrm{p}=0.0058$ ) (Figure 3 ).
Table 1. Relationship of podoplanin expression in CAFs with selected clinicopathological parameters. Significant $p$ values for the Fisher exact test are marked bold.

\begin{tabular}{|c|c|c|c|c|}
\hline \multirow{2}{*}{ Parameters } & \multirow{2}{*}{ No. (\%) } & \multicolumn{2}{|c|}{ Podoplanin CAFs - No. (\%) } & \multirow{2}{*}{$\mathrm{p}$ value } \\
\hline & & IRS 0-2 & IRS 3-12 & \\
\hline \multicolumn{5}{|l|}{ Age } \\
\hline$\leq 50$ & $35(33.7)$ & $12(34.2)$ & $23(65.8)$ & 0.4942 \\
\hline$>50$ & $69(66.3)$ & $18(26.1)$ & $51(73.9)$ & \\
\hline \multicolumn{5}{|l|}{ Menopause } \\
\hline Pre & $37(35.6)$ & $12(32.4)$ & $25(67.6)$ & 0.6521 \\
\hline Post & $67(64.4)$ & $18(26.8)$ & $49(73.2)$ & \\
\hline \multicolumn{5}{|l|}{ pT } \\
\hline pT1 & $61(58.6)$ & $23(37.7)$ & $38(62.3)$ & 0.0271 \\
\hline pT2-pT4 & $43(41.4)$ & $7(16.3)$ & $36(83.7)$ & \\
\hline \multicolumn{5}{|l|}{$\mathrm{pN}$} \\
\hline Negative & $54(51.9)$ & $17(31.5)$ & $37(68.5)$ & 0.6655 \\
\hline Positive & $50(48.1)$ & $13(26.0)$ & $37(74.0)$ & \\
\hline \multicolumn{5}{|l|}{ Grade } \\
\hline G1,G2 & $68(65.4)$ & $27(39.7)$ & $41(60.3)$ & 0.0006 \\
\hline G3 & $36(34.6)$ & $3(8.3)$ & $33(91.7)$ & \\
\hline \multicolumn{5}{|l|}{ ER } \\
\hline Positive & $84(80.8)$ & $27(32.1)$ & $57(67.9)$ & 0.1728 \\
\hline Negative & $20(18.2)$ & $3(15.0)$ & $17(85.0)$ & \\
\hline \multicolumn{5}{|l|}{ PR } \\
\hline Positive & $72(69.2)$ & $24(33.3)$ & $48(66.7)$ & 0.1625 \\
\hline Negative & $32(30.8)$ & $6(18.8)$ & $26(81.2)$ & \\
\hline \multicolumn{5}{|l|}{ HER2 } \\
\hline Positive & $17(16.3)$ & $3(17.6)$ & $14(82.4)$ & 0.3829 \\
\hline Negative & $87(83.7)$ & $27(31.0)$ & $60(69.0)$ & \\
\hline
\end{tabular}

IRS: immunoreactive score

Table 2. Distribution of analyzed IHC markers of angio- and lymphangiogenesis in the analyzed IDC patient cohort.

\begin{tabular}{lccc}
\hline IHC Marker & Cut-off value & $\begin{array}{c}\text { Low } \\
\text { expression } \\
\text { No. }(\%)\end{array}$ & $\begin{array}{c}\text { High } \\
\text { expression } \\
\text { No. }(\%)\end{array}$ \\
\hline VEGF-A & IRS 0-4 vs. 6-12 & $52(50.0)$ & $52(50.0)$ \\
VEGF-C & IRS 0-4 vs. 6-12 & $46(44.2)$ & $58(45.8)$ \\
VEGF-D & IRS 0-4 vs. 6-12 & $54(51.9)$ & $50(48.1)$ \\
Intratumoral CD31 MVC & $\leq 5 v s .>5$ & $49(47.1)$ & $55(52.9)$ \\
Peritumoral CD31 MVC & $\leq 6$ vs. $>6$ & $44(42.3)$ & $60(57.7)$ \\
Intratumoral D2-40 MVC & $\leq 2 v s .>2$ & $52(50.0)$ & $52(50.0)$ \\
Peritumoral D2-40 MVC & $\leq 4$ vs. $>4$ & $54(51.9)$ & $50(48.1)$ \\
Intratumoral Lyve-1 MVC & $\leq 1 v s .>1$ & $97(93.2)$ & $7(6.8)$ \\
Peritumoral Lyve-1 MVC & $\leq 2 v s .>2$ & $57(54.8)$ & $47(45.2)$ \\
\hline
\end{tabular}

IRS: immunoreactive score 

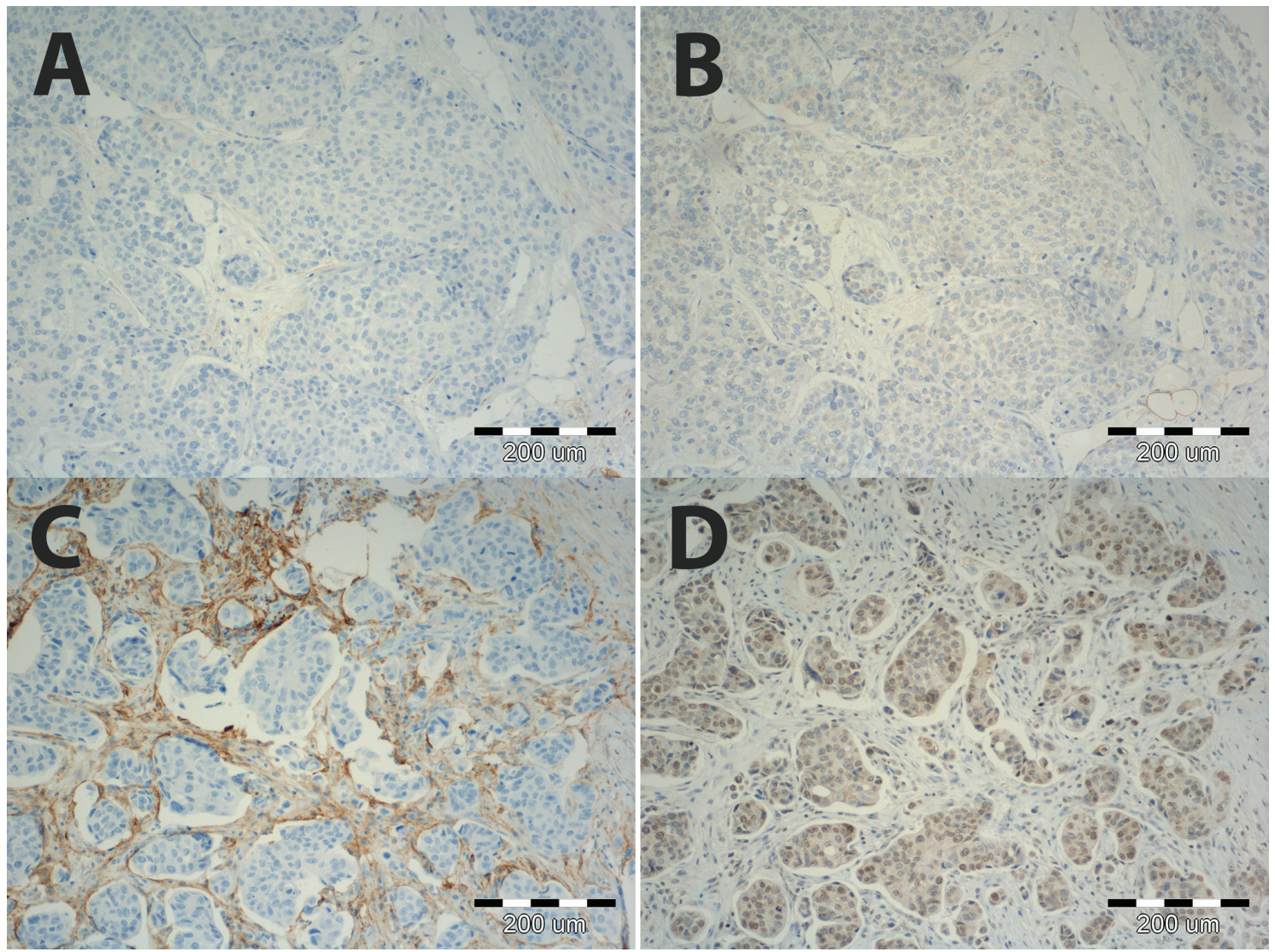

Figure 2. Serial sections of IDC immunostained for podoplanin in CAFs (A, C) and VEGF-C in cancer cells (B, D) corresponding to the noted positive correlation of both the analyzed markers. Cases characterized by low (A, B) and high (C, D) expression of these proteins. Magnification $\times 100$.

Medians of all the analyzed IHC markers were used to define the cut-off values for the univariate and multivariate survival analysis (Table 3). Mantel Cox test revealed that larger primary tumor size (pT2-pT4; $\mathrm{p}=0.0156)$, presence of lymph node metastasis $(p=0.0006)$, grade 3 (G3) of malignancy $(\mathrm{p}=0.0203)$ and high VEGF-C expression $(\mathrm{p}=0.0178)$ were associated with patients poor OS. These significant factors were entered in multivariate analysis. The Cox proportional hazards model revealed, that only the presence of lymph node metastasis was an independent marker of poor prognosis (Table 3).

\section{Discussion}

Recent studies identified podoplanin as a novel activity marker of tumor stroma of different malignancies [11-16, 31-33]. In majority of the examined tumors we and others have shown that podoplanin expression in CAFs was associated with patients poor outcome, but in some, e.g. squamous non-small cell lung cancer (SCC) and colorectal carcinoma predicted patients favourable prognosis [11-16, 31, 32]. Using IHC methods, Hoshino et al. demonstrated a positive correla- tion between podoplanin expression in CAFs and lymphatic vessel density [31]. Similar observations were noted in our recent study performed on 117 cases of IDC, although no such relationship was observed by Schoppmann et al. on a larger patients cohort $[12,13]$. In addition, podoplanin overexpression in SCC cell line EBC-1 led to the attenuation of prolymphangiogenic potential of these cells, but did not alter their potential of inducing tumor angiogenesis [25]. Such actions of podoplanin might be explained by the activation of c-jun- $\mathrm{NH}_{2}$-kinases (JNK) pathway in these cells after podoplanin overexpression. This led to the subsequent downregulation of VEGF-C expression in EBC-1 cells, which could be responsible for reduced lymphangiogenesis in the engrafted EBC-1-podoplanin-positive tumors [25]. In recent studies, VEGF-C was shown to induce the activity of the RhoA pathway, what resulted in formation of the capillary like structures of the HUVEC (Human Umbilical Vein Endothelial Cells) cells and points to possible proangiogenic actions of VEGF-C aside from its prolymphangiogenic potential $[34,35]$.

RhoA activity may be also regulated by podoplanin through its ERM (ezrin-radixin-moesin) binding domain, what results 

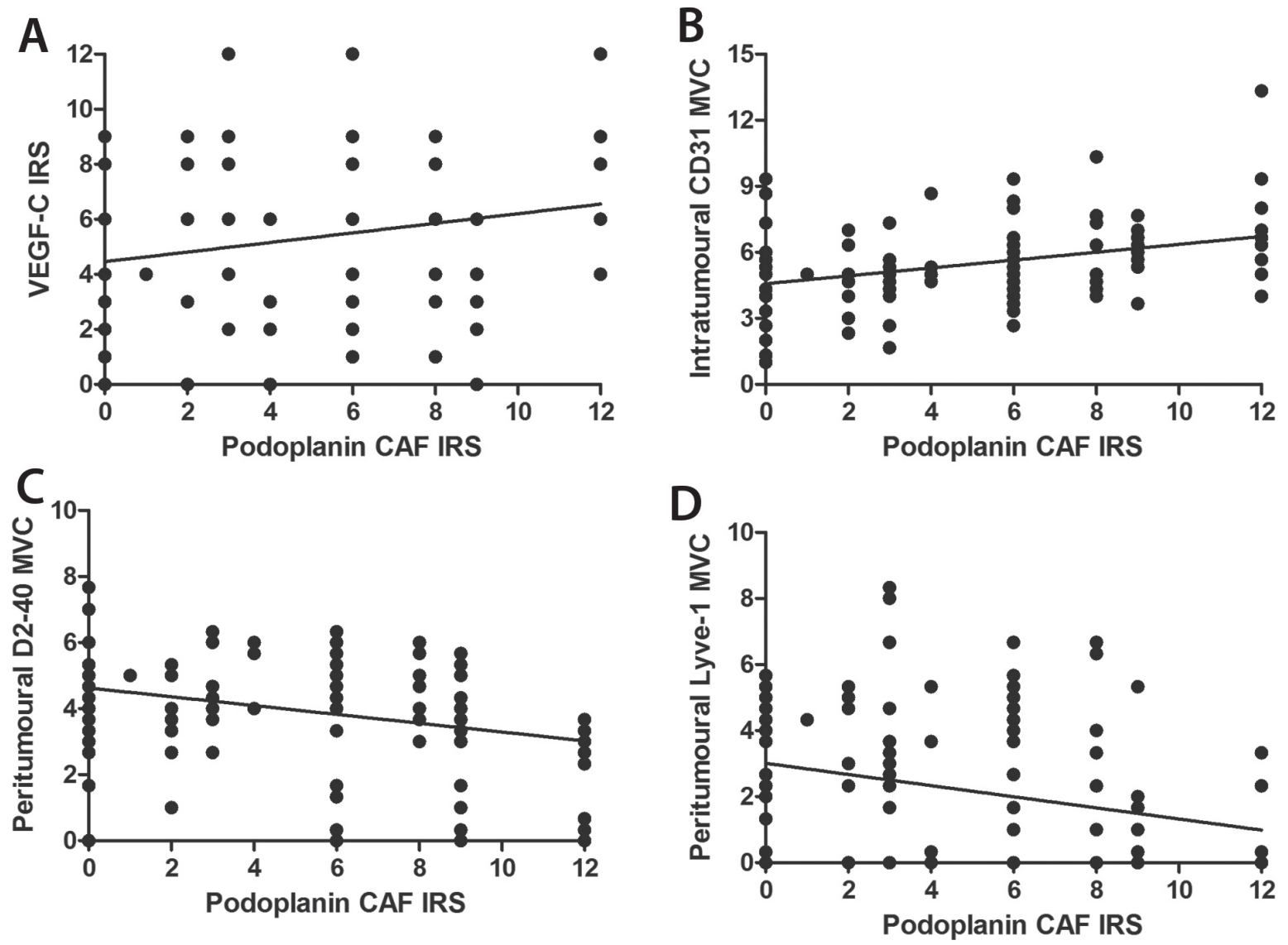

Figure 3. Significant correlations (Spearman correlation rank test) of podoplanin expression in CAFs with VEGF-C expression in cancer cells (A), intratumoral CD31 MVC (B), peritumoral D2-40 MVC (C) and peritumoral Lyve-1 MVC (D)

Table 3. Univariate (Mantel-Cox test) and multivariate (Cox proportional hazards model) overall survival analysis of 104 studied patients.

\begin{tabular}{|c|c|c|c|c|c|c|}
\hline \multirow[t]{2}{*}{ Clinicopathological parameter (cut-off) } & \multicolumn{3}{|c|}{ Univariate } & \multicolumn{3}{|c|}{ Multivariate } \\
\hline & HR & $95 \% \mathrm{CI}$ & $\mathrm{p}$ value & HR & $95 \% \mathrm{CI}$ & $\mathrm{p}$ value \\
\hline Age $(\leq 50 v s .>50)$ & 1.705 & $0.6857-4.237$ & 0.2510 & & & \\
\hline Menopausal status (Pre vs. Post) & 1.165 & $0.4709-2.881$ & 0.7414 & & & \\
\hline Tumor size (pT1 vs. pT2-pT4) & 3.145 & $1.242-7.964$ & 0.0156 & 1.650 & $0.6338-4.2965$ & 0.3048 \\
\hline Lymph node involvement $(\mathrm{N}-v s . \mathrm{N}+)$ & 4.745 & $1.959-11.49$ & 0.0006 & 4.868 & $1.379-17.174$ & 0.0138 \\
\hline Grade (G1, G2 vs. G3) & 3.092 & $1.192-8.022$ & 0.0203 & 1.818 & $0.7224-4.575$ & 0.2041 \\
\hline Podoplanin CAFs (0-2 vs. 3-12) & 2.082 & $0.7988-5.429$ & 0.1335 & & & \\
\hline VEGF-A (0-4 vs. 6-12) & 1.149 & $0.4760-2.772$ & 0.7576 & & & \\
\hline VEGF-C (0-4 vs. 6-12) & 2.911 & $1.203-7.047$ & 0.0178 & 2.669 & $0.8694-8.193$ & 0.0862 \\
\hline VEGF-D (0-4 vs. 6-12) & 1.419 & $0.5879-3.425$ & 0.4363 & & & \\
\hline Intratumoral CD31 MVC $(\leq 5$ vs. $>5)$ & 0.9621 & $0.3446-2.686$ & 0.7813 & & & \\
\hline Peritumoral CD31 MVC $(\leq 6$ vs. $>6)$ & 1.902 & $0.7884-4.591$ & 0.1525 & & & \\
\hline Intratumoral D2-40 MVC $(\leq 2 v s .>2)$ & 1.494 & $0.6189-3.605$ & 0.3721 & & & \\
\hline Peritumoral D2-40 MVC ( $\leq 4$ vs. $>4)$ & 0.8842 & $0.3660-2.136$ & 0.7845 & & & \\
\hline Intratumoral Lyve-1 MVC $(\leq 1$ vs. $>1)$ & 1.407 & $0.2581-7.666$ & 0.6932 & & & \\
\hline Peritumoral Lyve-1 MVC $(\leq 2 v s .>2)$ & 1.623 & $0.6719-3.923$ & 0.2817 & & & \\
\hline
\end{tabular}

HR: hazard-ratio; CI: confidence interval; MVC: microvessel count 
in EMT in the MDCK (Madin-Darby Canine Kidney) cells after podoplanin overexpression [23]. Similarly, Ito et al., demonstrated that introduction of podoplanin expression in human fibroblasts led to elevated RhoA levels, what resulted in enhanced tumor formation of A549 lung cancer cells, when both cell types were co-injected into SCID mice [36]. The above mentioned research demonstrate that actions of podoplanin may vary depending on its expression in various cell types and that this effect may be mediated by the expressions of RhoA and VEGF-C [23, 25, 36].

Using IHC method we have investigated the relationship between podoplanin expression and markers of angio- and lymphangiogenesis. In our study a positive correlation between podoplanin expression in CAFs and VEGF-C expression in IDC cancer cells was noted. Whether, induction of podoplanin expression is mediated via paracrine action of VEGF-C or an unidentified receptor remains to be clarified. To note, earlier in vitro studies reported a relationship between those two proteins in SCC EBC-1 cell line [25]. Moreover, podoplanin expression in CAFs or in EBC- 1 cells showed no impact on the expression levels of VEGF-A and VEGF-D, what is in agreement with our data obtained in this study performed on IDC tissues [25]. Cancer cells could stimulate podoplanin expression in CAFs via induction of RhoA activity by VEGF-C expression [34, 35]. In addition, VEGF-C was also shown to induce moesin expression, a protein of the ERM protein complex which also interacts with podoplanin, via RhoA/ROCK2 pathway in the cervical carcinoma SiHa cell line [35]. This pathway may be in part responsible for induction of podoplanin expression in CAFs of IDC by VEGF-C, but this hypothesis would require further research.

In contrast, to the study of Suzuki et al., we did not demonstrate associations with the intratumoral lymphatic MVC measured by the expression of Lyve-1 and D2-40 [25]. Interestingly, a weak negative correlation of podoplanin expression in CAFs with lymphatic MVC of both markers in the peritumoral area was observed. As the data obtained by us are based only on IHC, we may not confirm the hypothetical induction of podoplanin in CAFs by VEGF-C cancer cells expression as well as the observed negative correlation between podoplanin expression in CAFs and lymphatic MVC in the peritumoral compartment. To note, VEGF-C expression in the analyzed patient cohort did not show any impact on lymphatic MVC in both intra- and peritumoral compartments, although VEGF-C was shown to exert prolymphangiogenic properties in numerous tumors $[15,37]$.

We demonstrated a positive correlation between podoplanin expression in CAFs and MVC of CD31 positive vessels in the intratumoral compartment, what may support the thesis of local activity of podoplanin expressing CAFs on tumor vascularity. CAFs of different malignancies were shown to enhance tumor angiogenesis $[3,5,6,8]$.

Similar to our previous work and the work of Schoppmann et al., podoplanin expression in CAFs was associated with larger primary tumor size and higher malignancy grade [ $[12$, 13]. However, in this study we did not observe any relationship of podoplanin expression in CAFs with the presence of lymph node metastasis and patients overall survival. To better assess the interactions of podoplanin expression and expression of VEGFs in cancer cells, we utilized the scoring system based on reaction intensity and percentage of positive cells showing reaction product [28]. The use of the IRS scale for assessment of podoplanin expression and VEGFs expression in cancer cells allowed for their reliable comparison and statistical analysis. This relationship was the key point of research in this study. Nevertheless, use of different assessment scales could produce discrepant results due to cut-off values used in the statistical analysis. Until now, no consensus concerning the assessment methods of stromal markers was achieved, what might render some studies incomparable [11-16, 25, 31-33].

In summary, in this study we have showed that podoplanin expression in CAFs may have impact on angio- and lymphangiogenesis processes in IDC via induction of VEGF-C, but further research is required to define the interactions of these two proteins. In addition, international guidelines concerning stromal assessment scales should be corroborated, what would allow direct comparison of studies assessing stromal markers in different malignancies.

Acknowledgements: The authors thank Mrs Teresa Klepuszewszka, Ms Aleksandra Jethon and Mrs Aleksandra Piotrowska for their technical support.

This publication was supported by scientific grants: "Wrovasc - Integrated Cardiovascular Centre" project, co-financed by the European Regional Development Fund, within the Innovative Economy Operational Program, 2007-2013.

\section{References}

[1] HANAHAN D, WEINBERG RA. Hallmarks of cancer: the next generation. Cell 2011; 144: 646-74. http://dx.doi.org/10.1016/ j.cell.2011.02.013

[2] FOLKMAN J. Anti-angiogenesis: new concept for therapy of solid tumors. Ann Surg 1972; 175: 409-16. http://dx.doi. org/10.1097/00000658-197203000-00014

[3] ALLEN M, LOUISE JONES J. Jekyll and Hyde: the role of the microenvironment on the progression of cancer. J Pathol 2011; 223: 162-76. http://dx.doi.org/10.1002/path.2803

[4] LIOTTA LA, KOHN EC. The microenvironment of the tumor-host interface. Nature 2001; 411: 375-9. http://dx.doi. org/10.1038/35077241

[5] ORIMO A, GUPTA PB, SGROI DC, ARENZANA-SEISDEDOS F, DELAUNAY T et al. Stromal fibroblasts present in invasive human breast carcinomas promote tumor growth and angiogenesis through elevated SDF-1/CXCL12 secretion. Cell 2005; 121: 335-48. http://dx.doi.org/10.1016/ j.cell.2005.02.034

[6] BOIRE A, COVIC L, AGARWAL A, JACQUES S, SHERIFI $S$ et al. PAR1 is a matrix metalloprotease- 1 receptor that 
promotes invasion and tumorigenesis of breast cancer cells. Cell 2005; 120: 303-13. http://dx.doi.org/10.1016/ j.cell.2004.12.018

[7] STERNLICHT MD, LOCHTER A, SYMPSON CJ, HUEY B, ROUGIER JP et al. The stromal proteinase MMP3/stromelysin-1 promotes mammary carcinogenesis. Cell 1999; 98: 137-46. http://dx.doi.org/10.1016/S0092-8674(00)81009-0

[8] KALLURI R, ZEISBERG M. Fibroblasts in cancer. Nat Rev Cancer 2006; 6: 392-401. http://dx.doi.org/10.1038/nrc1877

[9] BREITENEDER-GELEFF S, SOLEIMAN A, KOWALSKI H, HORVAT R, AMANN G et al. Angiosarcomas express mixed endothelial phenotypes of blood and lymphatic capillaries: podoplanin as a specific marker for lymphatic endothelium. Am J Pathol 1999; 154: 385-94. http://dx.doi.org/10.1016/ S0002-9440(10)65285-6

[10] GANDARILLAS A, SCHOLL FG, BENITO N, GAMALLO C, QUINTANILLA M. Induction of PA2.26, a cell-surface antigen expressed by active fibroblasts, in mouse epidermal keratinocytes during carcinogenesis. Mol Carcinog 1997; 20: 10-8. http://dx.doi.org/10.1002/(SICI)1098-2744(199709)20:1<10:: AID-MC3>3.0.CO;2-M

[11] KAWASE A, ISHII G, NAGAI K, ITO T, NAGANO T et al. Podoplanin expression by cancer associated fibroblasts predicts poor prognosis of lung adenocarcinoma. Int J Cancer 2008; 123: 1053-9. http://dx.doi.org/10.1002/ijc.23611

[12] SCHOPPMANN SF, BERGHOFF A, DINHOF C, JAKESZ R, GNANT M, DUBSKY P, JESCH B, HEINZL H, BIRNER P. Podoplanin-expressing cancer-associated fibroblasts are associated with poor prognosis in invasive breast cancer. Breast Cancer Res Treat 2012; 134: 237-44. http://dx.doi. org/10.1007/s10549-012-1984-X

[13] PULA B, JETHON A, PIOTROWSKA A, GOMULKIEWICZ A, OWCZAREK T et al. Podoplanin expression by cancer-associated fibroblasts predicts poor outcome in invasive ductal breast carcinoma. Histopathology 2011; 59: 1249-60. http:// dx.doi.org/10.1111/j.1365-2559.2011.04060.x

[14] YAMANASHI T, NAKANISHI Y, FUJII G, AKISHIMAFUKASAWA Y, MORIYA Y et al. Podoplanin expression identified in stromal fibroblasts as a favorable prognostic marker in patients with colorectal carcinoma. Oncology 2009; 77: 53-62. http://dx.doi.org/10.1159/000226112

[15] AISHIMA S, NISHIHARA Y, IGUCHI T, TAGUCHI K, TAKETOMI A et al. Lymphatic spread is related to VEGF-C expression and D2-40-positive myofibroblasts in intrahepatic cholangiocarcinoma. Mod Pathol 2008; 21: 256-64. http:// dx.doi.org/10.1038/modpathol.3800985

[16] KITANO H, KAGEYAMA S, HEWITT SM, HAYASHI R, DOKI Y et al. Podoplanin expression in cancerous stroma induces lymphangiogenesis and predicts lymphatic spread and patient survival. Arch Pathol Lab Med 2010; 134: 1520-7.

[17] ZIMMER G, OEFFNER F, VON MESSLING V, TSCHERNIG T, GRONESS HJ et al. Cloning and characterization of gp36, a human mucin-type glycoprotein preferentially expressed in vascular endothelium. Biochem J 1999; 341 (Pt 2): 277-84. http://dx.doi.org/10.1042/0264-6021:3410277

[18] WICKI A, LEHEMBRE F, WICK N, HANTUSCH B, KERJASCHKI D et al. Tumor invasion in the absence of epithelial-mesenchymal transition: podoplanin-mediated remodeling of the actin cytoskeleton. Cancer Cell 2006; 9: 261-72. http://dx.doi.org/10.1016/j.ccr.2006.03.010

[19] CUENI LN, HEGYI I, SHIN JW, ALBINGER-HEGYI A, GRUBER $S$ et al. Tumor lymphangiogenesis and metastasis to lymph nodes induced by cancer cell expression of podoplanin. Am J Pathol 2010; 177: 1004-16. http://dx.doi.org/10.2353/ ajpath.2010.090703

[20] MARTIN-VILLAR E, FERNANDEZ-MUNOZ B, PARSONS M, YURRITA MM, MEGIAS D et al. Podoplanin associates with CD44 to promote directional cell migration. Mol Biol Cell 2010; 21: 4387-99. http://dx.doi.org/10.1091/mbc.E10$\underline{06-0489}$

[21] RAICA M, CIMPEAN AM, RIBATTI D. The role of podoplanin in tumor progression and metastasis. Anticancer Res 2008; 28: 2997-3006.

[22] WICKI A, CHRISTOFORI G. The potential role of podoplanin in tumor invasion. Br J Cancer 2007; 96: 1-5. http://dx.doi. org/10.1038/sj.bjc. 6603518

[23] MARTIN-VILLAR E, MEGIAS D, CASTEL S, YURRITA MM, VILARO S et al. Podoplanin binds ERM proteins to activate RhoA and promote epithelial-mesenchymal transition. J Cell Sci 2006; 119: 4541-53. http://dx.doi. org/10.1242/jcs.03218

[24] FERNANDEZ-MUNOZ B, YURRITA MM, MARTIN-VILLAR E, CARRASCO-RAMIREZ P, MEGIAS D et al. The transmembrane domain of podoplanin is required for its association with lipid rafts and the induction of epithelialmesenchymal transition. Int J Biochem Cell Biol 2011; 43: 886-96. http://dx.doi.org/10.1016/j.biocel.2011.02.010

[25] SUZUKI H, ONIMARU M, YONEMITSU Y, MAEHARA Y, NAKAMURA S et al. Podoplanin in cancer cells is experimentally able to attenuate prolymphangiogenic and lymphogenous metastatic potentials of lung squamoid cancer cells. Mol Cancer 2010; 9: 287. http://dx.doi.org/10.1186/1476-4598-9-287

[26] ELSTON CW, ELLIS IO. Pathological prognostic factors in breast cancer. I. The value of histological grade in breast cancer: experience from a large study with long-term follow-up. Histopathology 1991; 19: 403-10. http://dx.doi.org/10.1111/ j.1365-2559.1991.tb00229.x

[27] GOMULKIEWICZ A, PODHORSKA-OKOLOW M, SZULC R, SMORAG Z, WOJNAR A et al. Correlation between metallothionein (MT) expression and selected prognostic factors in ductal breast cancers. Folia Histochem Cytobiol 2010; 48: 242-8. http://dx.doi.org/10.2478/v10042-010-0011-5

[28] REMMELE W, STEGNER HE. [Recommendation for uniform definition of an immunoreactive score (IRS) for immunohistochemical estrogen receptor detection (ER-ICA) in breast cancer tissue]. Pathologe 1987; 8: 138-40.

[29] VERMEULEN PB, GASPARINI G, FOX SB, COLPAERT C, MARSON LP et al. Second international consensus on the methodology and criteria of evaluation of angiogenesis quantification in solid human tumors. Eur J Cancer 2002; 38: 1564-79. http://dx.doi.org/10.1016/S0959-8049(02)00094-1

[30] GOLDHIRSCH A, INGLE JN, GELBER RD, COATES AS, THURLIMANN B et al. Thresholds for therapies: highlights of the St Gallen International Expert Consensus on the pri- 
mary therapy of early breast cancer 2009. Ann Oncol 2009; 20: 1319-29. http://dx.doi.org/10.1093/annonc/mdp322

[31] HOSHINO A, ISHII G, ITO T, AOYAGI K, OHTAKI Y et al. Podoplanin-positive fibroblasts enhance lung adenocarcinoma tumor formation: podoplanin in fibroblast functions for tumor progression. Cancer Res 2011; 71: 4769-79. http://dx.doi. org/10.1158/0008-5472.CAN-10-3228

[32] ITO M, ISHII G, NAGAI K, MAEDA R, NAKANO Y et al. Prognostic impact of cancer-associated stromal cells in stage I lung adenocarcinoma patients. Chest 2012. http://dx.doi. org/10.1378/chest.11-2458

[33] CARVAlHO FM, ZAGANELli FL, ALMEIDA BG, GOES JC, BARACAT EC et al. Prognostic value of podoplanin expression in intratumoral stroma and neoplastic cells of uterine cervical carcinomas. Clinics (Sao Paulo) 2010; 65: 1279-83. http://dx.doi.org/10.1590/S1807$\underline{59322010001200009}$
[34] KUMAR B, CHILE SA, RAY KB, REDDY GE, ADDEPALLI $\mathrm{MK}$ et al. VEGF-C differentially regulates VEGF-A expression in ocular and cancer cells; promotes angiogenesis via RhoA mediated pathway. Angiogenesis 2011; 14: 371-80. http:// dx.doi.org/10.1007/s10456-011-9221-5

[35] HE M, CHENG Y, LI W, LIU Q, LIU J et al. Vascular endothelial growth factor $\mathrm{C}$ promotes cervical cancer metastasis via up-regulation and activation of RhoA/ROCK-2/moesin cascade. BMC Cancer 2010; 10: 170. http://dx.doi.org/10.1186/ 1471-2407-10-170

[36] ITO S, ISHII G, HOSHINO A, HASHIMOTO H, NERI S et al. Tumor promoting effect of podoplanin-positive fibroblasts is mediated by enhanced RhoA activity. Biochem Biophys Res Commun 2012. http://dx.doi.org/10.1016/j.bbrc.2012.04.158

[37] ADAMS RH, ALITALO K. Molecular regulation of angiogenesis and lymphangiogenesis. Nat Rev Mol Cell Biol 2007; 8: 464-78. http://dx.doi.org/10.1038/nrm2183 